\title{
WI - Call for Papers Heft 5/2011
}

\section{Die Zukunft der Telekommunikation}

DOI 10.1007/s11576-009-0203-9

\section{Herausgeber des Schwerpunktthemas}

\author{
Dr. Udo Bub \\ Deutsche Telekom Laboratories \& \\ European Center for Information and \\ Communication Technologies (EICT) \\ Ernst-Reuter Platz 7 \\ 10587 Berlin \\ Deutschland \\ udo.bub@telekom.de \\ Prof. Dr. Helmut Krcmar \\ Technische Universität München \\ Boltzmannstr. 3 \\ 85748 Garching \\ Deutschland \\ krcmar@in.tum.de \\ Prof. Dr. Dres. h. c. Arnold Picot \\ Ludwig-Maximilians-Universität \\ München \\ Ludwigstr. 28 \\ 80539 München \\ Deutschland \\ picot@Imu.de
}

This article is also available in English via http://www.springerlink.com and http://www.bise-journal.org: Bub U, Krcmar H, Picot A (2010) BISE - Call for Papers, Issue 5/2011. The Future of Telecommunications. Bus Inf Syst Eng. doi:10.1007/s12599-009-0082-z.

\section{Schwerpunktthema}

Die Telekommunikationsindustrie unterliegt seit zwei Dekaden in besonderem Maße einer Veränderung, hervorgerufen durch den Durchbruch der Architektur des Internets als Kommunikationsinfrastruktur und als Massenmedium sowie durch neue Generationen des Mobilfunks. Diese Dynamik wird sich fortsetzen: Neue Netztechno- logien (z. B. Next Generation Networks, Long Term Evolution) verändern Kostenund Marktstrukturen. Innovationszyklen für Produkte und Technologien werden kürzer, Wertschöpfungsketten verändern sich. So treten Ausrüster in den OnlineDienstemarkt ein (wie z. B. Nokia mit einem eigenen Diensteportal) oder bieten über so genannte Managed Services selber Netzwerkbetrieb an (wie z. B. Alcatel-Lucent). Klassische Betreiber hingegen beginnen, eigene Endgeräte zu spezifizieren; klassische Computerhersteller wie Apple brechen in den Telekommunikationsmarkt sowohl über Endgeräte als auch über neue Dienste ein. Als Ausdruck der Emanzipation von Diensten und Infrastruktur bieten zahlreiche neue Unternehmen (Google ist nur das prominenteste Beispiel) internetbasierte Telekommunikations- und Datendienstleistungen netzübergreifend und ohne eigene Infrastruktur an, so genannte Overthe-Top-Anbieter.

Betreiber stehen oftmals vor der Frage, ob sie ihre Zukunft im reinen Netzbetrieb gestalten sollen oder die Margen über neuartige netzspezifische Dienste (unter Verwendung von netzzentrischen Dienste-Enablern) bzw. netzunabhängige Dienste erzielen sollen. In jedem Falle gilt es, die internen betrieblichen Abläufe effektiv und effizient zu gestalten und dabei stärker zu automatisieren.

Weitere neue Herausforderungen bieten Chancen, bedürfen aber auch einer sehr sorgfältigen technoökonomischen Bewertung. So birgt die laufende Netzwerktransformation hin zu einer IPbasierten Infrastruktur der Next Generation Networks hohe Effizienzpotenziale, bedarf aber auch Investitionen in Milliardenhöhe bei vielfältigen Entscheidungsvarianten.

Die anstehende Anbindung von Glasfaseranschlüssen bis in die Haushalte (Fiber to the Home) wird nicht nur für Endanwender Bandbreiten von mehr als $100 \mathrm{Mbit} / \mathrm{s}$ ermöglichen, sondern auch Betreibern eine neue Gestaltung der Netzwerkarchitektur sowie der internen Geschäftsprozesse bis hin zu einer berührungslosen (d. h. voll automatisierten) Provisionierung bieten. Dieser gewaltige Ausbau wird nicht von ehemaligen Monopolanbietern (sog. Incumbents) alleine getätigt werden, sondern geteilt werden. Geschäftsmodelle der Infrastrukturanbieter werden eine gegenseitige Wholesale-Fähigkeit notwendig machen. Dies bedeutet, dass auch Incumbents künftig von anderen Betreibern Leitungskapazität in Zugangsnetzen anmieten werden, was bisher nicht der Fall war. Zudem zeigt sich, dass in einigen Regionen öffentliches Engagement z. B. in Form von Public-PrivatePartnerships erforderlich werden könnte, um eine flächendeckende Versorgung mit hochleistungsfähigen Breitbandzugängen gewährleisten zu können.

Das Schwerpunktthema adressiert technoökonomische Fragestellungen und Lösungen zu Marktstrategie, Produkten, Methoden und Prozessen sowie Technologien und Architekturen in der sich wandelnden Branche. Beiträge aus den entsprechenden Bereichen der Wirtschaftsinformatik, der Betriebswirtschaftslehre sowie der Informatik und Informations- und Nachrichtentechnik sind willkommen.

Dabei sind Beiträge aus Forschung und Praxis u. a. zu folgenden (gerne auch weiteren) Themenfeldern erwünscht:

- Markt, Produkte und Unternehmensstrategien

- Veränderung der Wertschöpfungsketten und Herausbildung neuartiger Wertschöpfungssysteme

- Geschäftsmodelle

- Konvergenzaspekte von Festnetz, Mobilfunk, IT, Internet und Medien

- Marktentwicklung Bit Pipe vs. Service Provider

- Mobile Internetdienste, -anwendungen und -produkte

- Diensteinnovationen

- Netzneutralität und offener Netzzugang vs. vertikale Integration von Diensten und Netzen 
- Telekommunikation als Enabler weiterer Branchen (Medien, Energie, Automotive, Gesundheit, Logistik, Finance)

- Methoden und Prozesse

- Referenzmodelle (z. B. eTOM, Anwendung von ITIL auf Telekommunikationsunternehmen)

- Geschäftsprozessautomatisierung

- Agile Methoden für Systemintegration, Entwicklung und Betrieb

- Kostenmodellierung und -schätzung für Entwicklung und Betrieb großer Netze, Modellierung von Capex (Capital Expenditure) und Opex (Operational Expenditure)

- Datenschutz und Datensicherheit, Netzwerksicherheit, Anwendungssicherheit

- Technologien und Architekturen

- Anwendungsarchitekturen (z. B. für mobile Endgeräte, Backend)

- Architekturen von Service-DeliveryPlattformen

- Breitbandarchitekturen und deren ökonomische Bewertung

- Architekturen für Heimvernetzung

- Netzwerkvirtualisierung, IT-Virtualisierung und Architekturen für Cloud-Computing, Telekommunikationsinfrastruktur von Rechenzentren

- Betriebliche Informationssysteme, BSS (Business Support Systems), OSS (Operations Support Systems)
- Netzwerktransformation und -migration von klassischen Telekommunikationsprotokollen hin zu IP, Next Generation (Mobile) Networks

- (Mobile) Betriebssysteme, OpenSource-Software (z. B. Android, Chrome OS)

\section{Einreichung von Beiträgen}

Bitte reichen Sie Beiträge für die $\mathrm{Ru}$ briken WI - Aufsatz und WI - State of the Art bis spätestens 2010-0901 über das Online-Begutachtungssystem (http://www.editorialmanager.com/ buis/) ein. Bitte beachten Sie die Hinweise zu formaler Gestaltung und Umfang von Beiträgen für die WIRTSCHAFTSINFORMATIK/Business \& Information Systems Engineering (BISE). Vollständige Beiträge sollten höchstens zehn Druckseiten umfassen; das entspricht 50.000 Zeichen einschließlich Leerzeichen, abzüglich 5.000 Zeichen je Seite an Bildern. Ausführliche Autorenrichtlinien stehen unter http://www. wirtschaftsinformatik.de zum Download bereit.

Eingereichte Beiträge werden anonymisiert von mehreren Gutachtern in einem doppelt-blinden Verfahren auf Relevanz, Originalität und fachliche Qualität beurteilt. Neben den Herausgebern des
Schwerpunktthemas und jenen der Zeitschrift wirken dabei weitere ausgewiesene internationale Persönlichkeiten aus Wissenschaft und Praxis mit.

Ergänzend $\mathrm{zu}$ den Aufsätzen sind auch weitere Einreichungen zum Schwerpunktthema willkommen.

Angenommene Beiträge erscheinen identisch in Deutsch und Englisch. Die deutschsprachige Fassung erscheint in WIRTSCHAFTSINFORMATIK, die englischsprachige in Business \& Information Systems Engineering (BISE). Angenommene Beiträge werden in enger Zusammenarbeit von Autoren und einem professionellen Übersetzerteam übersetzt.

\section{Zeitplan}

Einreichung von Beiträgen: 2010-09-01

Benachrichtigung der Autoren:

2010-11-01

Abschluss der ersten Überarbeitung:

2011-01-17

Benachrichtigung der Autoren:

2011-03-14

Ggf. Abschluss einer zweiten Überarbeitung (einsprachig): 2011-05-16

Ggf. Abschluss einer zweiten Überarbeitung (zweisprachig): 2011-06-13

Geplanter Erscheinungstermin Heft

5/2011: Oktober 2011. 